\title{
Original
}

\section{Effect of Methylprednisolone on Intravascular Coagulation and Edema Formation Induced by Venous Occlusion}

\author{
Koichiro ABE ${ }^{1)}$, Hiroshi Goto ${ }^{2)}$, Akira HaKoshima ${ }^{2)}$, Motonobu Kimura ${ }^{2)}$, \\ Kirk T. Benson ${ }^{2)}$ and Kasumi AraKaWa ${ }^{2)}$
}

\begin{abstract}
Cause and effect relations between intravascular coagulation and increased vascular endothelial permeability have recently been recognized. The purpose of this study was to investigate the effect of high dose methylprednisolone on tissue edema formation and coagulation in canine legs subjected to venous occlusion. Eight mongrel dogs received $30 \mathrm{mg} / \mathrm{kg}$ methylprednisolone administration intravenously. Eight additional days received normal saline as controls. Hind leg venous pressure was raised to $75 \mathrm{mmHg}$ continuously for 5 hours by a tourniquet around the thigh. Venous blood was sampled before tourniquet inflation, six times during tourniquet inflation, and $60 \mathrm{~min}$ after tourniquet ination. The sample blood was used for measurement of Sonoclot coagulation analysis, prothrombin time, and activated partial thromboplastin time. At each time of venous blood sampling, circumferences of 3 points of the distal part of the hind leg were measured to assess edema. Coagulation studies indicated initial hypercoagulability, and later hypocoagulability during venous occlusion in the control group. In the methylprednisolone-treated group, coagulation parameters remained unchanged throughout the study. Percent increases in circumferences as indices of edema formation were significantly less in the methylprednisolone-treated group than in the control group. The results suggest that inhibitory action of methylprednisolone on activation of the coagulation system secondary to venous occlusion may be a key factor in maintaining the integrity of endothelial permeability, resulting in less edema formation.
\end{abstract}

Key words: methylprednisolone, intravascular coagulation, edema formation, venous occlusion

\section{Introduction}

Pulmonary edema can occur as one of the complications of hemorrhagic shock and blood transfusion ${ }^{1,2)}$. One of the mechanisms of this type of edema is thought to be due to increased capillary permeability to proteins ${ }^{3)}$. The study by van der Zee and Malik ${ }^{4}$ demonstrated that only dogs that had pulmonary intravascular coagulation developed pulmonary edema after hemorrhagic shock. They suggested that pulmonary edema in their dog model may be due to activation of edematogenic cellular and humoral factors associated with intravascular coagulation. Such a relation between coagulation and tissue edema is also

\footnotetext{
1) Department of Plastic and Reconstructive Surgery, Showa University School of Medicine, 1-5-8 Hatonodai, Shinagawa-ku, Tokyo 142, Japan.

2) Department of Anesthesiology, University of Kansas, Medical Center, 39th and Rainbow Boulevard, Kansas City, Kansas 66103, U.S.A.
} 
suggested in patients with hereditary angioneurotic edema ${ }^{5)}$. It has been well recognized that venous occlusnoi can cause not only tissue edema but also activation of coagulation. In addition, high doses of corticosteroid have been found to reduce tissue edema formation ${ }^{6)}$ and attenuate coagulation ${ }^{7}$. These reports prompted us to investigate the effect of methylprednisolone on both blood coagulation and edema formation induced by venous occlusion.

\section{Materials and Methods}

A total of 16 mongrel dogs of either sex weighing 16 to $28 \mathrm{~kg}$ were used in this study. The dogs were anesthetized with $30 \mathrm{mg} / \mathrm{kg}$ sodium pentobarbital intravenously. They were then intubated to assure adequate airway passage and allowed to ventilate spontaneously. A sixteen-gauge IV catheter was placed in a branch of the lateral saphenous vein. Venous pressure was monitored through this catheter via a membrane transducer (Hewlett Packard, 1295A) connected to a polygraph (Hewlett Packard, 7758B).

A pediatric size tourniquet was applied to the thigh of a hind leg, and was connected to a Shook tourniquet unit. Eight dogs were pretreated with $30 \mathrm{mg} / \mathrm{kg}$ methylprednisolone (MPS) by intravenous injection. Eight additional dogs received normal saline as controls. After control sampling of venous blood, the tourniquet was inflated to maintain saphenous venous pressure at $75 \mathrm{mmHg}$ during the next 5 hours. No intravenous fluid was administered throughout the experiment. Venous blood was sampled from the saphenous venous catheter at 15, 30, 60, 120, 240 and $300 \mathrm{~min}$ after tourniquet inflation. After blood sample collection at $300 \mathrm{~min}$, the tourniquet was deflated, and the last blood sample was collected $60 \mathrm{~min}$ after tourniquet release. At each sampling $4 \mathrm{ml}$ of venous blood was withdrawn and immediately transferred to a plastic tube containing anticoagulant (3.8\% sodium citrate in 9:1 volume ratio).

A measured volume of $2 \mathrm{ml}$ was used for the Sonoclot whole blood coagulation analysis. The remaining $2 \mathrm{ml}$ was centrifuged at $3000 \mathrm{rpm}$, for $15 \mathrm{~min}$ at $4^{\circ} \mathrm{C}$, and the citrated plasma was saved for measurement of PT and PTT.

\section{Measurement of Whole Blood Coagulation}

The Sonoclot coagulation analyzer (Sienco, Inc., Colorado) is a proven device for measurement and recording of the entire clotting process of whole blood ${ }^{8,9)}$. This instrument has a continuously vibrating probe that is partially immersed into the blood sample. Any change in viscoelastic properties of the blood during coagulation is imposed upon the probe and converted to mechanical impedance. Here citrated blood $(0.4 \mathrm{ml})$ was placed in a plastic cuvet containing a stir bar (SonACT \#-800-0400, Sienco, Inc.) and was pretreated at $37^{\circ} \mathrm{C}$ for $3 \mathrm{~min}$, and $10 \mu \mathrm{l}$ of $0.5 \mathrm{M} \mathrm{CaCl}$ was then added to the citrated blood. The mixture was stirred for 5 seconds, and the probe was carefully lowered into the cuvet. At the same time, recording of the impedance change was started on the chart.

In the Sonoclot tracing, the initial horizontal line reflects that thrombin is being produced, but the viscoelasticity of the blood has not changed ("onset time"). Thus, a shorter onset time indicates a faster rate of thrombin generation. After the onset time has elapsed, the viscoelasticity of the blood starts to increase because of fibrin strand formation. Accordingly, the tracing shows an upstroke curve; the steepness of the curve is called the primary slope "rate" (units/min). The rate is therefore a reflection of how fast fibrin crosslinkages are being formed (Fig. 1).

2. Measurements of prothrombin time (PT) and activated partial thromboplastin time (PTT). 


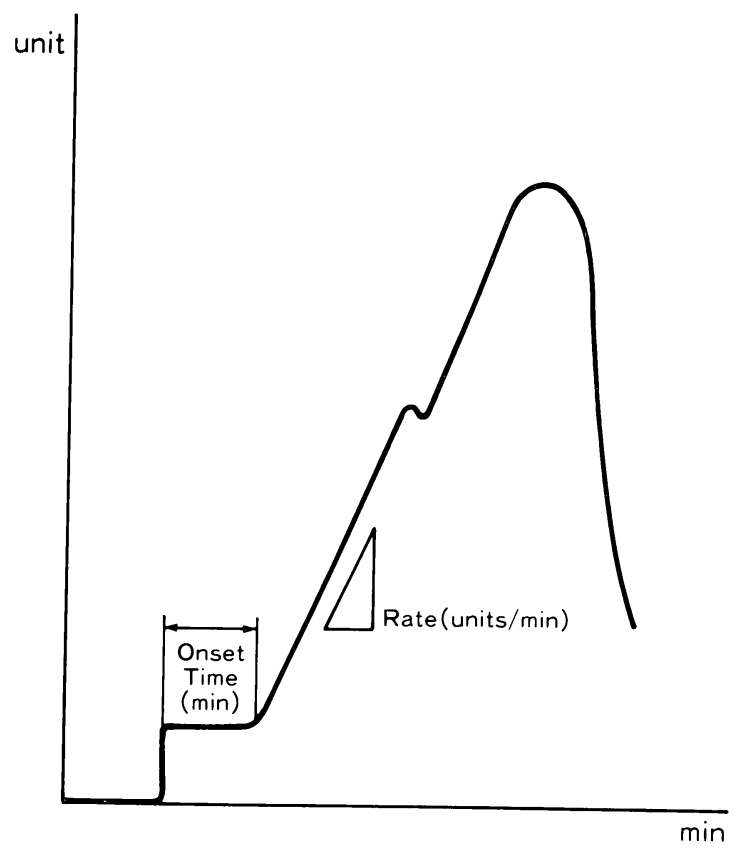

Fig. 1. Chart of Sonoclot coagulation analyzer.

PT was determined by the modified one-stage prothrombin time test using dried thromboplastin (Curtin Mathewson Scientific, Inc.). PTT was measured using Kontact (Curtin Mathewson Scientific, Inc.) as a reagent. PT and PTT were measured with duplicate determinations and the averages were used.

\section{Measurement of Edema Formation}

Edema formation (swelling) was measured as the change in hind leg circumference on three arbitrary lines as follows: 1) circumference of the line that passes through the carpometacarpal (CM) joint. 2) circumference of the line through the midpoint between the $\mathrm{CM}$ and the metacarpophalangeal (MP) joint. 3) circumference of the line that passes through the MP joint. Swelling was expressed as the percent increase in circumference.

Results were analyzed using analysis of variance followed by Duncan analysis for intergroup differences. A P value less than 0.05 was considered to be statistically significant.

\section{Results}

1. Sonoclot Whole Blood Coagulation Analysis

Figure 2 shows the changes in onset time and primary slope rate. Control onset time was $28.1 \pm 2.8(\mathrm{sec})($ mean $\pm \mathrm{SE}$ ), and rate was $35.5 \pm 3.2$ units $/ \mathrm{min}$. After venous occlusion was instituted by tourniquet inflation, onset time became significantly shorter and primary slope rate became significantly steeper in the control group. Later, during venous occlusion, these changes were reversed. Onset time was significantly prolonged and the primary slope rate became significantly less steep. These changes returned to the baseline values $60 \mathrm{~min}$ after turniquet deflation. In the MPS-treated group, neither onset time nor rate changed significantly throughout the venous occlusion. 

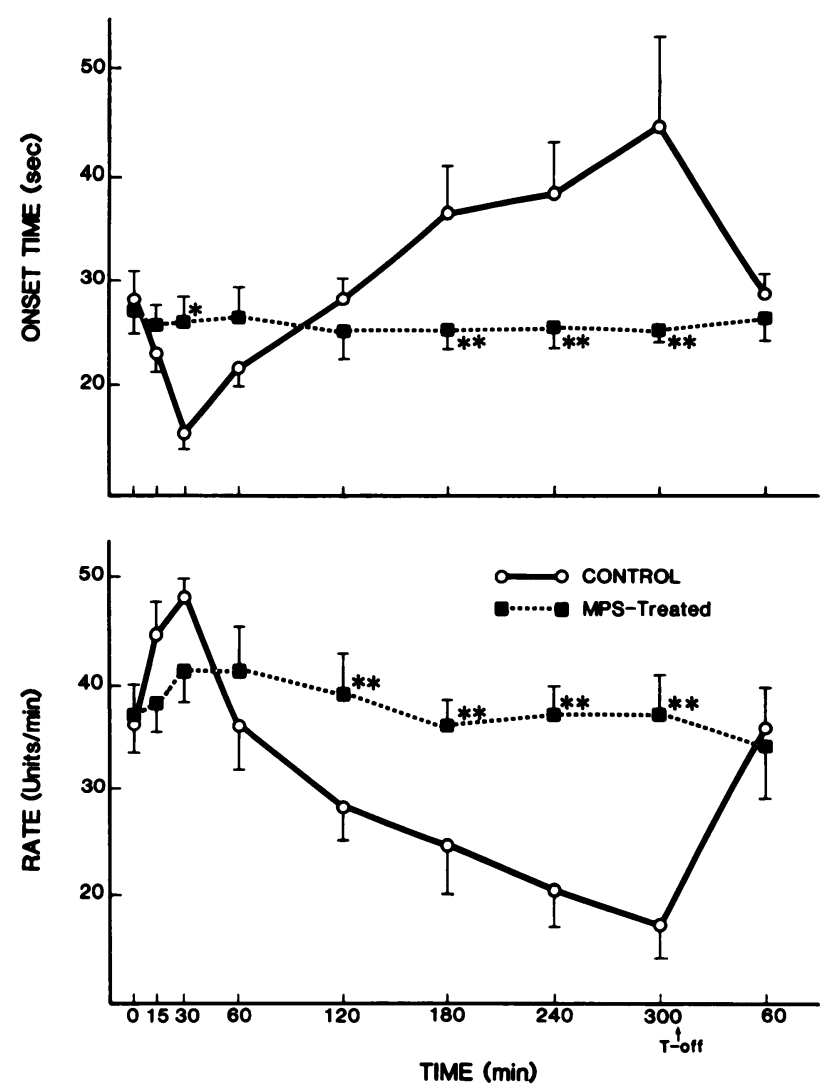

Fig. 2. Changes in onset time and rate in Sonoclot coagulation analysis before, during $(300 \mathrm{~min})$ and $60 \mathrm{~min}$ after venous occlusion. T-off; tourniquet deflated. ${ }^{*} \mathrm{p}<0.05$, $* * \mathrm{p}<0.01$ between control and MPS-treated groups.

\section{PT and PTT}

PT and PTT (Fig. 3) were shortened significantly after the venous occlusion was started in the control group. Later, during venous occlusion, PT and PTT values were significantly prolonged. In the MPS-treated group, PT and PTT value remained unchanged throughout the venous occlusion.

3. Edema formation

Circumferences increased steadily and significantly during the course of venous occlusion (Fig. 4). However, the increases in circumferences were significantly less in the MPS-treated group at all periods of measurement during venous occlusion. After tourniquet deflation, circumferences decreased precipitously, but still remained elevated compared to the baseline values.

\section{Discussion}

Glucocorticoids have been used clinically to treat tissue edema although their effectiveness is sometimes equivocal ${ }^{10)}$. The present study, however, demonstrated the efficacy of methylprednisolone, one of the glucocorticoids, in decreasing the degree of edema due to venous 


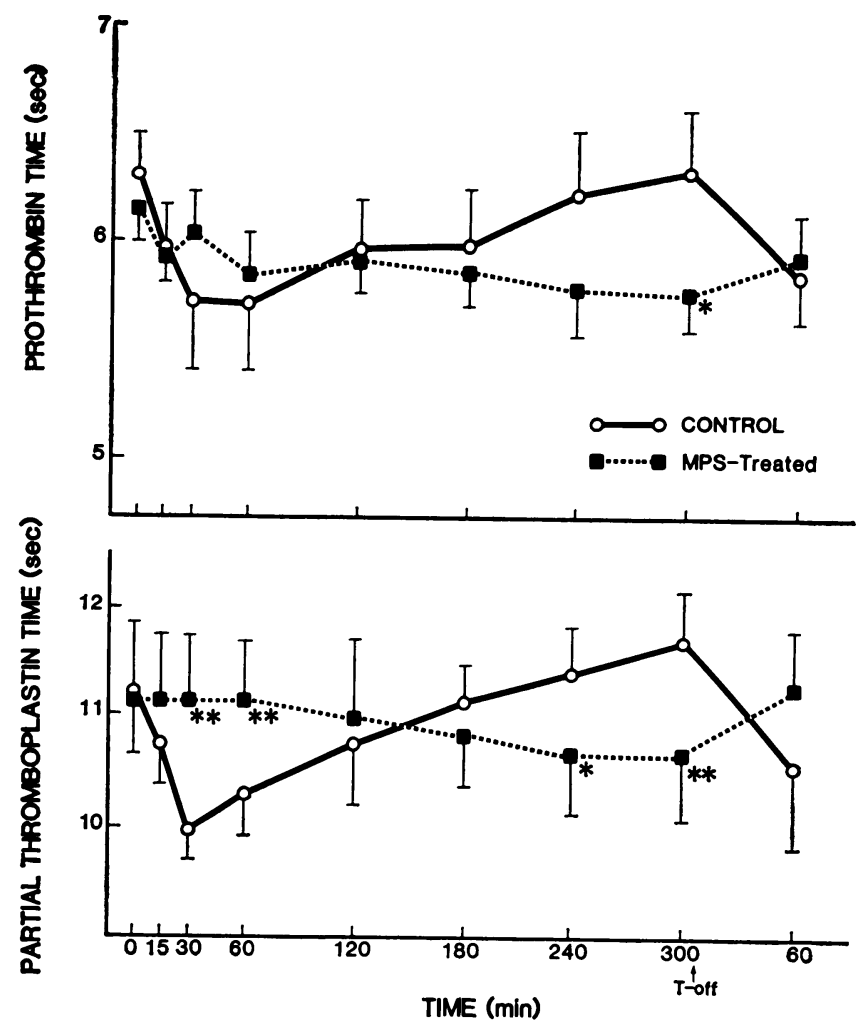

Fig. 3. Changes in prothrombin time and activated partial thromboplastin time. ${ }^{*} \mathrm{p}<0.05$, $* * \mathrm{p}<0.01$ between the two groups.

occlusion in canine legs. It is generally accepted that tissue edema develops mainly due to either increased intravascular hydrostatic pressure or increased endothelial permeability secondary to endothelial injury of various etiologies. It is apparent in our study that edema developed due to the increased transcapillary filtration secondary to the raised venous pressure. Since MPS should not affect edema formation due to the raised venous pressure, an observed difference in the degree of edema between two groups is most likely due to the development of permeability edema in the control group.

Egeberg $^{11)}$ showed that venous pressures up to $65 \mathrm{mmHg}$ could induce activation of the coagulation system and the coagulation system was markedly altered when venous pressure reached $90 \mathrm{mmHg}$ in the human forearm. Nillson and Robertson ${ }^{12)}$ and Porani et al. ${ }^{13)}$ reported that prolonged venous occlusion induces not only activation of coagulation, but also fibrinolysis. Larsson and Risberg ${ }^{14)}$ indicated that the venous occlusion-induced fibrinolysis is due to the release of plasminogen activators from the vascular wall. Therefore, the observed initial hypercoagulability and later hypocoagulability in the control group is reflecting, at least in part, change of the coagulation system, fibrinolytic system and coagulation factors. As is known, the coagulation and fibrinolytic systems form biological links with the kallikreinkinin system and the complement system ${ }^{15,16)}$. Formation of bradykinin is one of the most important factors of edema formation ${ }^{1 i}$ ). In addition, change in the clotting system can be 


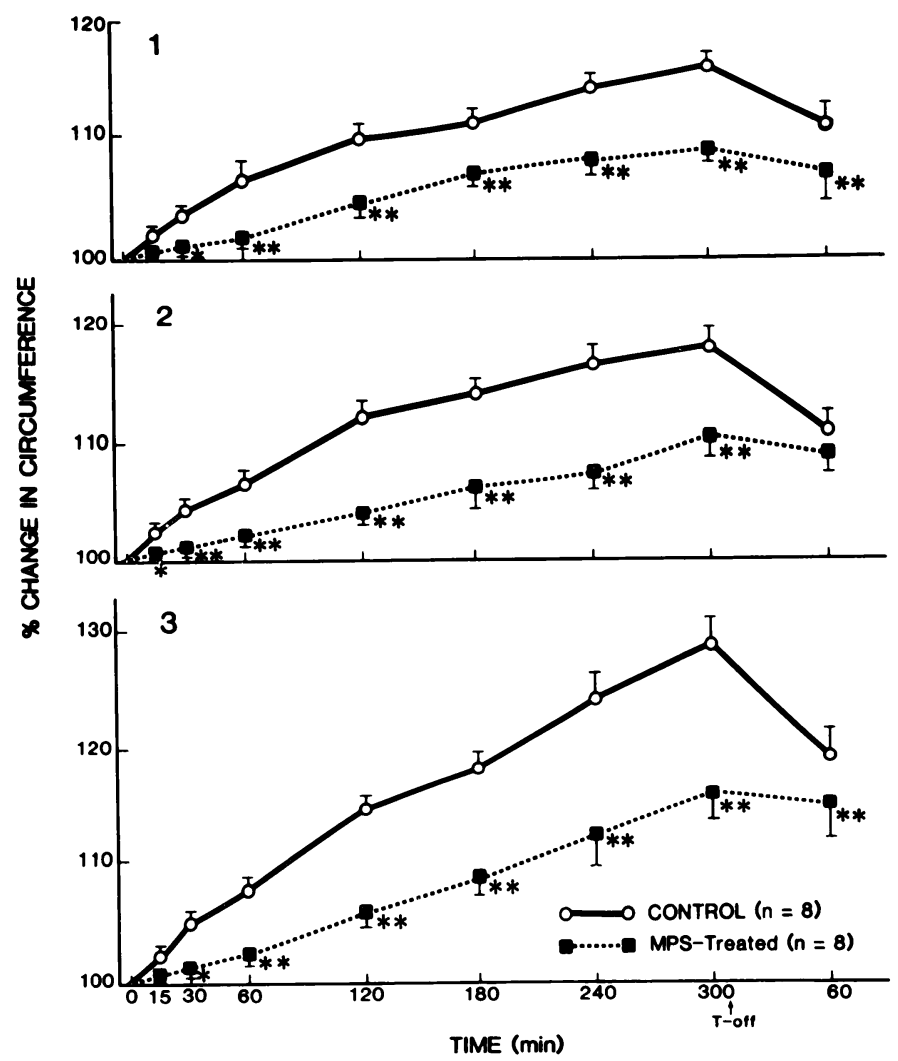

Fig. 4. Percent change in circumference of hind leg.

1) around the carpometacarpal (CM) joint,

2) around the point between $\mathrm{CM}$ joint and metacarpophalangeal (MP) joint,

3) around the MP joint. ${ }^{*} \mathrm{p}<0.05,{ }^{*} \mathrm{p}<0.01$ between the two groups.

accompanied by the release of edemagenic humoral factors such as histamine ${ }^{4)}$. Furthermore, intravascular coagulation results in the activation of leukocytes which can cause vascular injury leading to tissue edema ${ }^{18)}$. It is, therefore, speculated that venous occlusion of $75 \mathrm{mmHg}$ venous pressure produced tissue edema not only by increased microvascular hydrostatic pressure but also by increased vascular endothelial permeability particularly in the control group.

In the control group, blood initially became hypercoagulable and it later became hypocoagulable during venous occlusion. Such changes were entirely abolished by pretreatment with MPS. From these results, we can speculate that MPS has a protective effect on the coagulation system. The mechanism by which MPS interferes with coagulation changes is still not completely known. Latour and Renaud reported that corticosteroids inhibit the consumption of Hageman Factor (in rabbits) ${ }^{19)}$ and platelets (in rats) ${ }^{20)}$ after endotoxin administration. Nelson and Taylor ${ }^{21)}$ and Rosenberg et al. ${ }^{22)}$ reported that corticosteroids in vitro prevent endotoxin-induced platelet aggregation and decrease the availability of Platelet Factor 3, a phospholipid procoagulant.

The degree of edema was also significantly less in the MPS-treated group. Since venous 
hydrostatic pressure was the same in both groups, the reduction of edema in the MPS group was probably due to prevention of the increaes in vascular permeability. Thus, the results indirectly support the study by van der Zee and Malik ${ }^{4)}$ in which a causal relation was suggested between the coagulation derangement and increased vascular endothelial permeability.

In summary, our results showed that the degree of edema caused by venous occlusion in the hind leg of the dog was significantly reduced by pretreatment with methylprednisolone. It can be speculated that the protective effect of MPS on the coagulation system may be a key factor in maintaining the integrity of the endothelial permeability, resulting in less edema during venous occlusion.

\section{References}

1) Moss GS: Pulmonary involvement in hemorrhagic shock. Ann Rev Med, 23: 201-228 (1972)

2) Northrup WF and Humphrey EW: The effect of hemorrhagic shock on pulmonary vascular permeability to plasma proteins. Surgery, 83: 264-273 (1978)

3) Tood TRJ, Baile E and Hoog JC: Pulmonary capillary permeability during hemorrhagic shock. J Appl Physiol, 45: 298-306 (1978)

4) van der Zee $\mathbf{H}$ and Malik AB: Relationship of pulmonary edema after hemorrhagic shock to intravascular coagulation. Circ Shock, 10: 91-100 (1983)

5) Witzke G, Bork K, Benes $P$ and Bockers $M$ : Hereditary angioneurotic oedema and blood coagulation: Interaction between Cl-esterase-inhibitor and thə activation factors of the proteolitic enzyme systems. Klin Wochenschr, 6: 1131-1135 (1983)

6) Skijelbred $P$ and Sokken P: Reduction of the pain and swelling by a corticosteroid injection 3 hours after surgery. Eur J Clin Pharmacol, 23: 141-146 (1982)

7) Hardaway RM and Williams $\mathrm{CH}$ : Influence of steroids on hemorrhagic and traumatic shock. J Trauma, 27: 667-670 (1987)

8) Von Kaulla KN, Ostendort P and Von Kaulla K: The impedance machine: a new bedside coagulation recording device. J Med, 6: 73-87 (1975)

9) Chandler ML and Schmer G: Evaluation of a dynamic plasma. Clin Chem, 32: 505-507 (1986)

10) Sibbald WJ, Driedger AA, Finley RJ, Holliday RN, Austin TA, Petrakos A and Power J: Highdose corticosteroids in the treatment of pulmonary microvascular injury. Ann NY Acad Sci, 384: 496-516 (1982)

11) Egeberg O: The effect of venous congestion on the blood clotting system. Scan J Clin Lab Invest, 15: $20-28$ (1963)

12) Nillson IM and Robertson B: Effect of venous occlusion on coagulation and fibrinolytic components in normal subjects. Thromb Diath Haemorrh, 20: 397-408 (1968)

13) Ponari O, Pini M, Poti R and Dettori AG: Correlation between changes induced by venous occlusion on factor VIII-Von Willebrand factor components and fibrinolytic activity. Haemostasis, 14: 179-183 (1984)

14) Larsson $\mathrm{J}$ and Risberg B: Fibrinolytic activity in human legs in tourniquet ischemia. Thromb Res, 11: 817-825 (1977)

15) Voger $R$ and Zickgraf-Rudel G: Evaluation of the role of kinins in experimental, pathological and clinical conditions: the therapeutic use of kallikrein inhibitor. In: Handbook of Experimental Pharmacology, Erdos EH (Eds), Springer-Verlag, Berlin, Heidelberg, New York, pp 550-578 (1970)

16) Cullman W, Kovary PM, Muller N and Dick W: Complement, coagulation and fibrinolytic parameters in hereditary angioedema (HAE). Clin Exp Immunol, 49: 618-622 (1982)

17) Malik AB, Lee $\mathrm{BC}$ and van der Zee $\mathrm{H}$ : Mechanism of pulmonary edema induced microembolization in dogs. Circ Res, 42: 72-79 (1978)

18) Malik AB, Johnson A and Tahamont MV: Mechanism of lung vascular injury after intravascular coagulation. Ann NY Acad Sci, 384: 213-234 (1982)

19) Latour JG and Renaud S: Prevention by glucocorticoids of various blood parameter changes following surgical intervention and endotoxin administration in the rat. Can Med Assoc J, 100: 2026 (1969)

20) Latoru JG, Prejean JB and Margarethen W: Corticosteroids and the generalized Shwartzman re- 
action. Mechanism of sensitization in the rat. Am J Pathol, 65: 189-200 (1971)

21) Nelson WR and Taylor GA: In vitro inhibition endotoxin-induced platelet aggregation with hydrocortisone sodium succinate (Solu-Coref ${ }^{\circledR}$ ). Scand J Haematol, 15: 35-44 (1975)

22) Rosenberg A, Saundres WJ and Hawiger J: Influence of methylprednisolone on human platelet function. Inhibition of aggregation, serotonin release, and clot promoting phospholipid irrespective of membrane receptors involved. Clin Res, 24: 318 (1976) (Supplement)

[Received December 25, 1990: Accepted January 25, 1991] 